\title{
SPECTRAL MEASURES ON SPACES NOT CONTAINING $\ell^{\infty}$
}

\author{
by T. A. GILLESPIE
}

(Received 19th September 1979)

The property of weak sequential completeness plays a special role in the theory of Boolean algebras of projections and spectral measures on Banach spaces. For instance, if $X$ is a weakly sequentially complete Banach space, then

(i) every strongly closed bounded Boolean algebra of projections on $X$ is complete (3, XVII.3.8, p. 2201); from which it follows easily that

(ii) every spectral measure on $X$ of arbitary class $(\Sigma, \Gamma)$, where $\Sigma$ is a $\sigma$-algebra of sets and $\Gamma$ is a total subset of the dual space of $X$, is strongly countably additive; and hence that

(iii) every prespectral operator on $X$ is spectral.

(See also (1, Theorem 6.11, p. 165) for (iii).)

In fact, a necessary and sufficient condition for (i) to be valid is that $X$ should not contain a subspace isomorphic to the sequence space $c_{0}(4)$. In the present note, it is shown that properties (ii) and (iii) are equivalent and that a necessary and sufficient condition for one, and hence both, to hold is that $X$ should not contain a subspace isomorphic to the sequence space $\ell^{\infty}$. This answers affirmatively a question raised by $\mathrm{H}$. R. Dowson, who asked whether every prespectral operator on a separable Banach space is necessarily spectral. Actually, a slight refinement (Theorem 2 below) gives a separability condition which is both necessary and sufficient for an individual prespectral operator to be spectral.

For the most part, the terminology and notation will be as in (1). Throughout, $X$ is a complex Banach space with dual space $X^{*},\langle x, \phi\rangle$ denotes the value of $\phi \in X^{*}$ at $x \in X$, and $L(X)$ is the algebra of all bounded linear operators on $X$. The closed subspace generated by a subset $A$ of $X$ is denoted by $\operatorname{clm} A$ and the spectrum of a bounded linear operator by $\mathrm{Sp}(T)$.

Given a $\sigma$-algebra $\Sigma$ of subsets of a set $\Omega$ and a total subset $\Gamma$ of $X^{*}$, a spectral measure of class $(\Sigma, \Gamma)$ on $X$ is an identity preserving Boolean algebra homomorphism $E$ of $\Sigma$ onto a bounded Boolean algebra of projections on $X$ such that, for all $x$ in $X$ and all $\gamma$ in $\Gamma,\langle E(\cdot) x, \gamma\rangle$ is countably additive on $\Sigma$. It is a consequence of a theorem of Pettis (2, IV.10.1, p. 318) that a spectral measure $E(\cdot)$ of class $\left(\Sigma, X^{*}\right)$ is strongly countably additive (i.e. $E(\cdot) x$ is countably additive in the norm topology for each $x$ in $X)$. The $\sigma$-algebra of Borel subsets of the complex plane $C$ is denoted by $\Sigma_{p}$. An operator $T$ in $L(X)$ is prespectral if there is a total subset $\Gamma$ of $X^{*}$ and a spectral measure $E(\cdot)$ on $X$ of class $\left(\Sigma_{p}, \Gamma\right)$ such that

$$
E(\sigma) T=T E(\sigma) \text { and } \operatorname{Sp}(T \mid E(\sigma) X) \subset \bar{\sigma}
$$


for all $\sigma \in \Sigma_{p}$. In this case, $T$ is said to be prespectral of class $\Gamma$ and $E(\cdot)$ is called a resolution of the identity of class $\Gamma$ for $T$. A prespectral operator has a unique resolution of the identity of a given class, but may have distinct resolutions of the identity of (necessarily) different classes (1, Chapter 5 ). $T$ is spectral if there is a strongly countably additive spectral measure $E(\cdot)$ on $X$, defined on $\Sigma_{p}$ and satisfying (1). Note that, using Pettis's theorem as above or by (1, Theorem 6.5, p. 161), an operator on $X$ is spectral if and only if it is prespectral of class $X^{*}$. Furthermore, it follows from the uniqueness theorem for resolutions of the identity of a given class that, given a prespectral operator $T$ with resolution of the identity $E(\cdot)$ of class $\Gamma, T$ is spectral if and only if $E(\cdot)$ is strongly countably additive.

Theorem 1. The following statements are equivalent.

(i) $X$ does not contain a subspace isomorphic to $\ell^{\infty}$.

(ii) Every spectral measure on $X$ of arbitrary class $(\Sigma, \Gamma)$, where $\Sigma$ is a $\sigma$-algebra of sets and $\Gamma$ is a total subset of $X^{*}$, is strongly countably additive.

(iii) Every prespectral operator on $X$ is spectral.

Proof. To prove (i) $\Rightarrow$ (ii), assume (i) holds and let $E(\cdot)$ be a spectral measure on $X$ of class $(\Sigma, \Gamma)$, where $\Sigma$ is a $\sigma$-algebra of subsets of a set $\Omega$ and $\Gamma$ is a total subset of $X^{*}$. Suppose that $E(\cdot)$ is not strongly countably additive. Then there is a sequence $\left\{\sigma_{n}\right\}$ of mutually disjoint elements of $\Sigma$ and an element $x$ in $X$.such that the series.

$$
\sum_{n=1}^{\infty} E\left(\sigma_{n}\right) x
$$

does not converge in norm to $y=E\left(\bigcup_{n=1}^{\infty} \sigma_{n}\right) x$. If this series converged in norm to some element $z$ of $X$, we would have

$$
\langle z, \gamma\rangle=\sum_{n=1}^{\infty}\left\langle E\left(\sigma_{n}\right) x, \gamma\right\rangle=\langle y, \gamma\rangle
$$

for all $\gamma \in \Gamma$ and hence $z=y$ since $\Gamma$ is total. This contradiction shows that the series (2) does not converge in norm. Hence there exist $\varepsilon>0$ and increasing sequences $\{n(k)\}$, $\{m(k)\}$ of positive integers with $n(k)<m(k)<n(k+1)$ and

$$
\left\|\sum_{n=n(k)}^{m(k)} E\left(\sigma_{n}\right) x\right\| \geqq \varepsilon
$$

for $k=1,2, \ldots$. Let

$$
\tau_{k}=\sigma_{n(k)} \cup \ldots \cup \sigma_{m(k)}
$$

for $k=1,2, \ldots$. Then $\left\{\tau_{k}\right\}$ is a sequence of mutually disjoint elements of $\Sigma$ such that

$$
\left\|E\left(\tau_{k}\right) x\right\| \geqq \varepsilon
$$

for all $k$.

Given an element $\alpha=\left\{\alpha_{k}\right\}$ in $\ell^{\infty}$, let $f_{\alpha}: \Omega \rightarrow C$ be defined by

$$
f_{\alpha}=\sum_{k=1}^{\infty} \alpha_{k} \chi_{k}
$$


where $\chi_{k}$ is the characteristic function of $\tau_{k}$. Note that this series converges pointwise on $\Omega$ since the $\tau_{k}$ 's are disjoint and that $f_{\alpha}$ is a bounded measurable function on $(\Omega, \Sigma)$. Let

$$
\theta \alpha=\left\{\int f_{\alpha}(\lambda) E(d \lambda)\right\} x \quad\left(\alpha \in \ell^{\infty}\right),
$$

the integral being defined as in $\left(1\right.$, p. 120). Then $\theta$ is a linear mapping of $\ell^{\infty}$ into $X$ such that

$$
\begin{aligned}
\|\theta \alpha\| & \leqq 4 M \sup \left\{\left|f_{\alpha}(\lambda)\right|: \lambda \in \Omega\right\}\|x\| \\
& =4 M\|x\|\|\alpha\|_{\infty}
\end{aligned}
$$

for all $\alpha \in \ell^{\infty}$, where

$$
M=\sup \{\|E(\sigma)\|: \sigma \in \Sigma\}
$$

Fixing $\alpha=\left\{\alpha_{k}\right\} \in \ell^{\infty}$, we have

$$
\begin{aligned}
M\|\theta \alpha\| & \geqq\left\|E\left(\tau_{k}\right) \theta \alpha\right\| \\
& =\left\|\left\{\int \chi_{k}(\lambda) f_{\alpha}(\lambda) E(d \lambda)\right\} x\right\| \\
& =\left|\alpha_{k}\right|\left\|E\left(\tau_{k}\right) x\right\| \\
& \geqq \varepsilon\left|\alpha_{k}\right|
\end{aligned}
$$

for each positive integer $k$. Hence

$$
\|\theta \alpha\| \geqq \varepsilon M^{-1}\|\alpha\|_{\infty} \quad\left(\alpha \in \ell^{\infty}\right) .
$$

Thus $\theta$ is bicontinuous and its range is a subspace of $X$ which is isomorphic to $\ell^{\infty}$. This contradicts (i) and hence establishes (i) $\Rightarrow$ (ii).

The implication (ii) $\Rightarrow$ (iii) is clear. To prove (iii) $\Rightarrow$ (i) we show that, if $X$ has a subspace isomorphic to $\ell^{\infty}$, then there is a non-spectral prespectral operator on $X$. Suppose then that $X$ contains a subspace isomorphic to $\ell^{\infty}$. It is convenient to identify this subspace with $\ell^{\infty}$, so that $\ell^{\infty}$ is actually contained algebraically in $X$. Since $\ell^{\infty}$ is injective (6, p. 105), there is a closed subspace $Y$ of $X$ such that

$$
X=\ell^{\infty} \oplus Y \text {. }
$$

It is known that there exist prespectral operators on $\ell^{\infty}$ which are not spectral. An example of such an operator is given, for instance, in (1, pp. 144 et seq.). (It is not explicitly stated in (1) that the prespectral operator presented there is not spectral, but this follows from the fact that it has a resolution of the identity which is not strongly countably additive.) Let $S$ be such a non-spectral prespectral opeator on $\ell^{\infty}$, with resolution of the identity $E(\cdot)$ of class $\Gamma$, and define $T$ in $L(X)$ by

$$
T=S \oplus 0
$$

relative to the decomposition (3). Identifying $X^{*}$ with $\left(\ell^{\infty}\right)^{*} \oplus Y^{*}$ in the natural way, it is readily verified that $T$ is prespectral of class $\Gamma \oplus Y^{*}$, with resolution of the identity $F(\cdot)$ given by

$$
F(\sigma)=E(\sigma) \oplus \delta(\sigma) I_{Y} \quad\left(\sigma \in \Sigma_{p}\right)
$$

relative to (3), where $\delta$ is the probability measure on $\boldsymbol{C}$ with unit mass at the point 0 
and $I_{Y}$ is the identity operator on $Y$. Noting that $F(\cdot)$ is not strongly countably additive, it follows that $T$ is not spectral.

Corollary. If $X$ is either weakly sequentially complete or separable, then statements (ii) and (iii) of Theorem 1 are valid.

Proof. A closed subspace of a weakly sequentially complete (resp. separable) Banach space is weakly sequentially complete (resp. separable). Since $\ell^{\infty}$ is neither weakly sequentially complete nor separable, the result follows immediately from Theorem 1.

Remarks. 1. Theorem 1 has been stated for complex Banach spaces because the theory of spectral operators is usually developed in that context. However, it is clear that (i) and (ii) are equivalent in the real case, provided $\ell^{\infty}$ is interpreted appropriately.

2. The example of a prespectral operator on $\ell^{\infty}$ cited in the proof of Theorem 1 has two distinct resolutions of the identity. It follows that each of the statements (i)-(iii) in Theorem 1 is equivalent to the following.

(iv) Every prespectral operator on $X$ has a unique resolution of the identity.

It would be of interest to know whether there is a result for individual prespectral operators corresponding to (iii) $\Leftrightarrow$ (iv). That is, is it true that an individual prespectral operator is spectral if and only if it has a unique resolution of the identity?

3. Note that, in the proof of (i) $\Rightarrow$ (ii), the existence of the total set $\Gamma$ was used only in one place. It is natural to ask whether there are circumstances in which every finitely additive spectral meaure on $X$, for which no countable additivity assumption whatsoever is made, is in fact strongly countably additive. However, no such result is possible if $X$ is non-zero. To see this, take $\Omega$ to be the positive integers $N$ and let $\Sigma$ be the $\sigma$-algebra of all subsets of $\Omega$. Let $\phi$ be a non-zero multiplicative linear functional on $\ell^{\infty}$ which is not a coordinate functional, so that $\phi$ corresponds to a point of $\beta \mathbf{N} \backslash \mathbf{N}$, and let

$$
E(\sigma)=\phi\left(\chi_{\sigma}\right) I
$$

$(\sigma \in \Sigma)$,

where $I$ is the identity operator on $X$ and $\chi_{\sigma}$ is the characteristic function of $\sigma$. Then $E(\cdot)$ is a finitely additive spectral measure, but there exists no non-zero $\gamma$ in $X^{*}$ such that $\langle E(\cdot) x, \gamma\rangle$ is countably additive for all $x$ in $X$.

The result of Theorem 1 can be used to give a necessary and sufficient condition for a prespectral operator to be spectral as follows.

Theorem 2. Let $T$ be a prespectral operator on $X$, with resolution of the identity $E(\cdot)$ of class $\Gamma$. Let

$$
M_{E}(x)=\operatorname{clm}\left\{E(\sigma) x: \sigma \in \Sigma_{p}\right\}
$$

for $x \in X$. Then $T$ is spectral if and only if $M_{E}(x)$ is separable for every $x \in X$.

Proof. Suppose firstly that $M_{E}(x)$ is separable for every $x$ in $X$. Fix $x \in X$ and let $\Gamma_{0}$ denote the restriction of $\Gamma$ to $M_{E}(x)$, a total subset of $M_{E}(x)^{*}$. Note that $M_{E}(x)$ is $E(\cdot)$-invariant and that the restriction of $E(\cdot)$ to $M_{E}(x)$ is a spectral measure of class $\Gamma_{0}$. 
By the above corollary, separability implies that this restriction is strongly countably additive and, since $x$ is arbitrary, it follows that $E(\cdot)$ is strongly countably additive. Hence $T$ is a spectral operator.

Conversely, let $T$ be spectral. Then $E(\cdot)$ is strongly countably additive. Let $\Sigma_{0}$ be a countable base for the topology of $\boldsymbol{C}$ and let $\Sigma_{1}$ be the collection of finite unions of sets in $\Sigma_{0}$. It will be shown that

$$
M_{E}(x)=\operatorname{clm}\left\{E(\sigma) x: \sigma \in \Sigma_{1}\right\}
$$

for each $x \in X$. Since $\Sigma_{1}$ is countable, it will follow that each space $M_{E}(x)$ is separable as required.

Fix $x \in X$ and let $\phi \in X^{*}$ satisfy

$$
\langle E(\sigma) x, \phi\rangle=0 \quad\left(\sigma \in \Sigma_{1}\right) .
$$

Then the positive and negative parts, $\mu^{+}$and $\mu^{-}$respectively, of the real part of the complex measure $\langle E(\cdot) x, \phi\rangle$ agree on each set in $\Sigma_{1}$ and hence on each open set, since every open set in $C$ is the union of an increasing sequence of sets in $\Sigma_{1}$. Using the regularity of $\mu^{+}$and $\mu^{-}\left(5\right.$, Theorem $G$, p. 228), it follows that $\mu^{+}=\mu^{-}$and hence that the real part of $\langle E(\cdot) x, \phi\rangle$ is the zero measure. Similarly, the imaginary part of $\langle E(\cdot) x, \phi\rangle$ vanishes identically and so

$$
\langle E(\sigma) x, \phi\rangle=0 \quad\left(\sigma \in \Sigma_{p}\right) .
$$

The Hahn-Banach theorem now gives (4) and completes the proof.

\section{REFERENCES}

(1) H. R. Dowson, Spectral theory of linear operators (Academic Press, London, 1978).

(2) N. Dunford and J. T. Schwartz, Linear operators, Part I: General theory (Wiley, New York, 1958).

(3) N. Dunford and J. T. SChwartz, Linear operators, Part III: Spectral operators (Wiley, New York, 1971).

(4) T. A. GILlespie, Strongly closed bounded Boolean algebras of projections, Glasgow Math. J. 22 (1981), 73-75.

(5) P. R. Halmos, Measure theory (van Nostrand, Princeton, 1950).

(6) J. Lindenstrauss and L. Tzafriri, Classical Banach spaces I (Springer-Verlag, Berlin, 1977).

Department of Mathematics

James Clerk Maxwell Bullding

The King's BuILdings

EDINBURGH EH9 3JZ 